\title{
Turán numbers for 3-uniform linear paths of length 3
}

\author{
Eliza Jackowska
}

A. Mickiewicz University

Poznań, Poland

elijac@amu.edu.pl

\author{
Joanna Polcyn
}

A. Mickiewicz University

Poznań, Poland

joaska@amu.edu.pl
Andrzej Ruciński*

A. Mickiewicz University
Poznań, Poland

rucinski@amu.edu.pl

Submitted: June 11, 2015; Accepted: Apr 24, 2016; Published: May 13, 2016

Mathematics Subject Classifications: 05D05, 05C65, 05B07

\begin{abstract}
In this paper we confirm a special, remaining case of a conjecture of Füredi, Jiang, and Seiver, and determine an exact formula for the Turán number $\operatorname{ex}_{3}\left(n ; P_{3}^{3}\right)$ of the 3 -uniform linear path $P_{3}^{3}$ of length 3 , valid for all $n$. It coincides with the analogous formula for the 3-uniform triangle $C_{3}^{3}$, obtained earlier by Frankl and Füredi for $n \geqslant 75$ and Csákány and Kahn for all $n$. In view of this coincidence, we also determine a 'conditional' Turán number, defined as the maximum number of edges in a $P_{3}^{3}$-free 3 -uniform hypergraph on $n$ vertices which is not $C_{3}^{3}$-free.
\end{abstract}

\section{Introduction}

A $k$-uniform hypergraph (or $k$-graph, for short) is an ordered pair $H=(V, E)$, where $V$ is a finite set and $E \subseteq\left(\begin{array}{l}V \\ k\end{array}\right)$ is a family of $k$-element subsets of $V$. We often identify $H$ with $E$, for instance, writing $|H|$ for the number of edges in $H$. Given a positive integer $n$ and a family of $k$-graphs $\mathcal{F}$, we say that a $k$-graph $H$ is $\mathcal{F}$-free if $H$ contains no member of $\mathcal{F}$ as a subhypergraph. The Turán number $\operatorname{ex}_{k}(n ; \mathcal{F})$ is defined as the maximum number of edges in an $\mathcal{F}$-free $k$-graph on $n$ vertices. We set $\operatorname{ex}_{3}(0 ; \mathcal{F})=0$ for convenience.

An $n$-vertex $k$-graph $H$ is called extremal with respect to $\mathcal{F}$ if $H$ is $\mathcal{F}$-free and $|H|=$ $\operatorname{ex}_{k}(n ; \mathcal{F})$. We denote by $\operatorname{Ex}_{k}(n ; \mathcal{F})$ the set of all, pairwise non-isomorphic $n$-vertex $k$ graphs which are extremal with respect to $\mathcal{F}$. If $\mathcal{F}=\{F\}$, then we write $F$-free instead of $\{F\}$-free and write $\operatorname{ex}_{k}(n ; F)$, and $\operatorname{Ex}_{k}(n ; F)$ instead of $\operatorname{ex}_{k}(n ;\{F\})$ and $\operatorname{Ex}_{k}(n ;\{F\})$.

A linear path $P_{m}^{k}$ is a $k$-graph with $m$ edges $e_{1}, \ldots, e_{m}$ such that $\left|e_{i} \cap e_{j}\right|=0$ if $|i-j|>1$ and $\left|e_{i} \cap e_{j}\right|=1$ if $|i-j|=1$ (see Fig.1 for $P_{3}^{3}$ ). Füredi, Jiang, and Seiver [7] have determined $\operatorname{ex}_{k}\left(n ; P_{m}^{k}\right)$ for all $k \geqslant 4, m \geqslant 1$, and sufficiently large $n$. In particular, their result for $m=3$ states that $\operatorname{ex}_{k}\left(n ; P_{3}^{k}\right)=\left(\begin{array}{l}n-1 \\ k-1\end{array}\right)$. They conjectured that this formula remains valid in

\footnotetext{
*Research supported by the Polish NSC grant 2014/15/B/ST1/01688.
} 
the case $k=3$ too. This conjecture was confirmed by Kostochka, Mubayi, and Verstraete in [11] for all $m \geqslant 4$ and large $n$. Moreover, it is possible to deduce the sole remaining case $k=m=3$ from Theorem 6.2 in [11], by using a standard stability argument, but, again, for large $n$ only.



Figure 1: The linear path $P_{3}^{3}$

In this paper we prove two theorems. Even though the 3 -uniform length 3 case was implicit in [11], our main result determines the Turán number for 3-uniform linear path of length 3 , together with a unique extremal 3-graph, for all $n$.

Let $K_{n}^{k}$ stand for the complete $k$-graph with $n$ vertices, that is, one with $\left(\begin{array}{l}n \\ k\end{array}\right)$ edges. Note that when $n<k$ this is just a set of $n$ isolated vertices. A star is a hypergraph containing a vertex which belongs to all of its edges. An $n$-vertex $k$-uniform star with $\left(\begin{array}{l}n-1 \\ k-1\end{array}\right)$ edges is called full and denoted by $S_{n}^{k}$. By $F \cup H$ we denote the union of vertex disjoint copies of $k$-graphs $F$ and $H$.

\section{Theorem 1.}

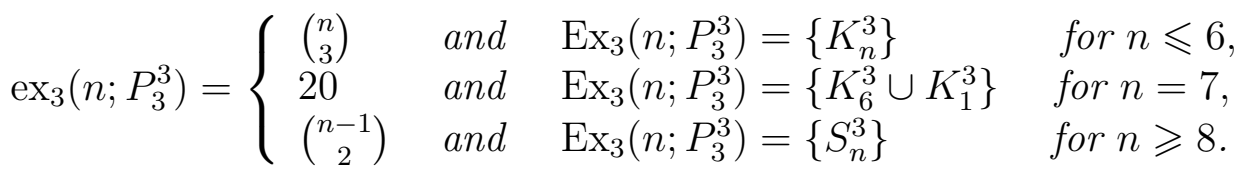

The proof of Theorem 1 relies on a similar result for 3-uniform linear cycles, or triangles. Let $C_{3}^{3}$ be the triangle defined as a 3-graph on 6 vertices $a, b, c, d, e, f$ and with 3 edges $\{a, b, c\},\{c, d, e\}$, and $\{e, f, a\}$. It was proved in [6] that $\operatorname{ex}_{3}\left(n ; C_{3}^{3}\right)=\left(\begin{array}{c}n-1 \\ 2\end{array}\right)$ for all $n \geqslant 75$. This has been later extended by Csákány and Kahn [3] to cover all $n$.

Theorem $2([6,3])$. For $n \geqslant 6, \operatorname{ex}_{3}\left(n ; C_{3}^{3}\right)=\left(\begin{array}{c}n-1 \\ 2\end{array}\right)$. Moreover, for $n \geqslant 8, \operatorname{Ex}_{3}\left(n ; C_{3}^{3}\right)=$ $\left\{S_{n}^{3}\right\}$.

Theorem 2 is the starting point of our proof of Theorem 1. Indeed, we show that having a triangle in a 3-graph with at least $\left(\begin{array}{c}n-1 \\ 2\end{array}\right)$ edges leads to the existence of a copy of $P_{3}^{3}$. In fact, it has turned out that the presence of $C_{3}^{3}$ pushes down the number of edges a $k$-graph may have without containing a copy of $P_{3}^{3}$. Motivated by this phenomenon, we also determine the largest number of edges in an $n$-vertex $P_{3}^{3}$-free 3 -graph, $n \geqslant 6$, which contains a triangle. We denote this 'conditional' Turán number by $\operatorname{ex}_{3}\left(n ; P_{3}^{3} \mid C_{3}^{3}\right)$ and the corresponding extremal family by $\operatorname{Ex}_{3}\left(n ; P_{3}^{3} \mid C_{3}^{3}\right)$. Our second result expresses $\operatorname{ex}_{3}\left(n ; P_{3}^{3} \mid C_{3}^{3}\right)$ in terms of the ordinary Turán numbers $\operatorname{ex}_{3}\left(n ; P_{3}^{3}\right)$.

Theorem 3. For $n \geqslant 6$,

$$
\operatorname{ex}_{3}\left(n ; P_{3}^{3} \mid C_{3}^{3}\right)=20+\operatorname{ex}_{3}\left(n-6 ; P_{3}^{3}\right) .
$$


Moreover, $\operatorname{Ex}_{3}\left(n ; P_{3}^{3} \mid C_{3}^{3}\right)=\left\{K_{6}^{3} \cup H_{n-6}\right\}$, where $\left\{H_{n-6}\right\}=\operatorname{Ex}_{3}\left(n-6, P_{3}^{3}\right)$, that is, the sole element of $\operatorname{Ex}_{3}\left(n ; P_{3}^{3} \mid C_{3}^{3}\right)$ is the disjoint union of $K_{6}^{3}$ and the unique extremal $P_{3}^{3}$-free 3-graph on $n-6$ vertices.

Theorem 3, combined with Theorem 1, yields also the exact value of $\operatorname{ex}_{3}\left(n ; P_{3}^{3} \mid C_{3}^{3}\right)$. For brevity, we state it for $n \geqslant 14$ only.

Corollary 1. For $n \geqslant 14$,

$$
\operatorname{ex}_{3}\left(n ; P_{3}^{3} \mid C_{3}^{3}\right)=20+\left(\begin{array}{c}
n-7 \\
2
\end{array}\right) \quad \text { and } \quad \operatorname{Ex}_{3}\left(n ; P_{3}^{3} \mid C_{3}^{3}\right)=\left\{K_{6}^{3} \cup S_{n-6}^{3}\right\} .
$$

Our last result follows rather from the proof of Theorem 3 than from the theorem itself. Let $\operatorname{ex}_{3}^{\text {con }}\left(n ; P_{3}^{3} \mid C_{3}^{3}\right)$ be defined as $\operatorname{ex}_{3}\left(n ; P_{3}^{3} \mid C_{3}^{3}\right)$, but where the maximum is taken over all connected graphs.

Corollary 2. For $n \geqslant 9$,

$$
\operatorname{ex}_{3}^{c o n}\left(n ; P_{3}^{3} \mid C_{3}^{3}\right)=3 n-8
$$

Remark 1 (Disjoint unions of $\boldsymbol{P}_{\mathbf{3}}^{\mathbf{3}}$ ). For a positive integer $s$, let $s F$ denote the vertexdisjoint union of $s$ copies of a hypergraph $F$. Bushaw and Kettle [2] determined, for large $n$, the Turán number $\operatorname{ex}_{k}\left(n ; s P_{m}^{k}\right)$, but only for those instances for which the Turán number $\operatorname{ex}_{k}\left(n ; P_{m}^{k}\right)$ had been known (they used induction on $\left.s\right)$. In particular, they have shown, for large $n$, that if $\operatorname{ex}_{3}\left(n ; P_{3}^{3}\right)=\left(\begin{array}{l}n \\ 3\end{array}\right)-\left(\begin{array}{c}n-1 \\ 3\end{array}\right)$, then $\operatorname{ex}_{3}\left(n ; s P_{3}^{3}\right)=\left(\begin{array}{l}n \\ 3\end{array}\right)-\left(\begin{array}{c}n-2 s+1 \\ 3\end{array}\right)$, providing also the unique extremal 3-graph, which happens to be the same as that for $M_{2 s}^{3}$, the matching of size $2 s$ (see [4]). By proving Theorem 1, we have, at the same time, verified the latter formula unconditionally.

\section{Preliminaries}

In what follows $H$ is always a $P_{3}^{3}$-free 3 -graph with $V(H)=V$ and $|V|=n \geqslant 7$, containing a copy $C$ of the triangle $C_{3}^{3}$. Let

$$
U=V(C), \quad U=U_{1} \cup U_{2}, \quad \text { where } \quad U_{1}=\left\{y_{1}, y_{2}, y_{3}\right\}, \quad U_{2}=\left\{x_{1}, x_{2}, x_{3}\right\},
$$

and

$$
C=\left\{\left\{x_{i}, y_{j}, x_{k}\right\}:\{i, j, k\}=\{1,2,3\}\right\},
$$

so that for $i=\{1,2\}, U_{i}$ is the set of vertices of degree $i$ in $C$ (see Fig. 2).

Further, let

$$
W=V \backslash U=\left\{w_{1}, \ldots, w_{s}\right\}, \quad|W|=s=n-6 .
$$

We split the set of edges of $H$ into three subsets (see Fig. 3),

$$
H[U]=H \cap\left(\begin{array}{c}
U \\
3
\end{array}\right), \quad H[W]=H \cap\left(\begin{array}{c}
W \\
3
\end{array}\right) \quad \text { and } \quad H(U, W)=H \backslash(H[U] \cup H[W]) .
$$






Figure 2: The triangle $C_{3}^{3}$

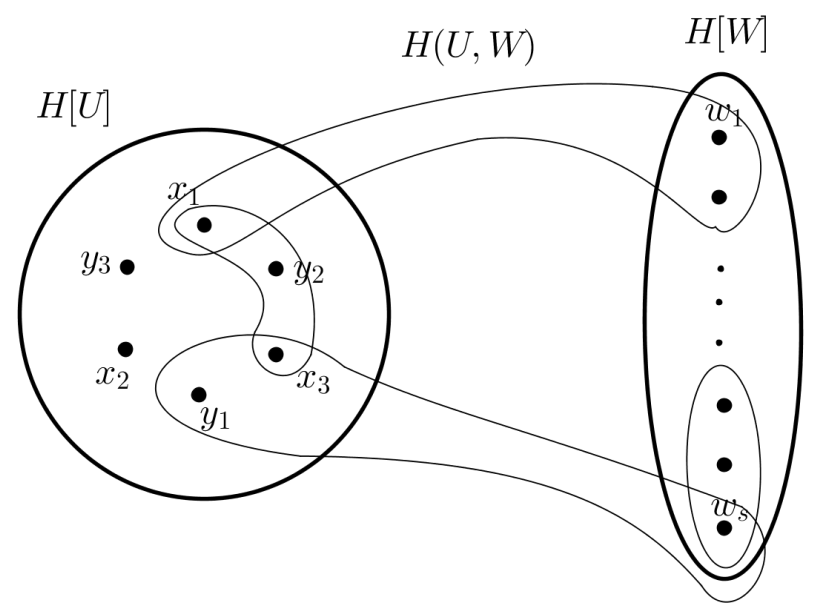

Figure 3: The partition of the set of edges of $H$

Let us also define two sets of triples (which are not necessarily edges of $H$ ):

$T_{1}=\left\{\left\{x_{i}, y_{i}, w_{l}\right\}: 1 \leqslant i \leqslant 3,1 \leqslant l \leqslant s\right\}, \quad T_{2}=\left\{\left\{x_{i}, x_{j}, w_{l}\right\}: 1 \leqslant i<j \leqslant 3,1 \leqslant l \leqslant s\right\}$

(see Fig. 4) and set

$$
T=T_{1} \cup T_{2} .
$$

We begin with several simple observations all of which can be verified by inspection. The first three have been already made in [9]. First of them says that although, in principle, $H(U, W)$ may consist of edges having one vertex in $U$ (and two in $W$ ), the assumption that $H$ is $P_{3}^{3}$-free makes it impossible. For the same reason, out of the potential edges with two vertices in $U$ (and one in $W$ ), only those listed in $T$ can actually occur in $H$.

Fact $1([9]$, Facts 1-3).

$$
H(U, W)=H \cap T
$$



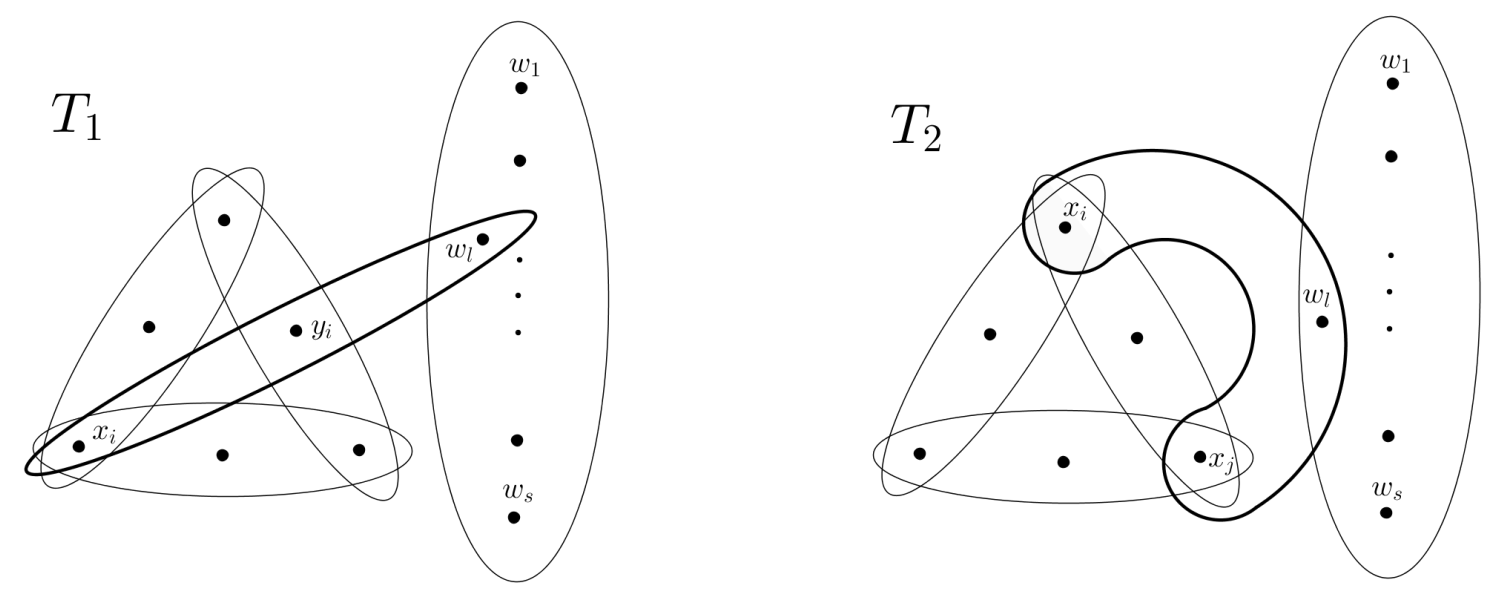

Figure 4: The edges in sets $T_{1}$ and $T_{2}$ are shaded

Next, we observe that if an edge from $T$ and another one from $\left(\begin{array}{c}W \\ 3\end{array}\right)$ have a common vertex, then, together with an edge of $C$, they form a $P_{3}^{3}$ (see Fig. 5).
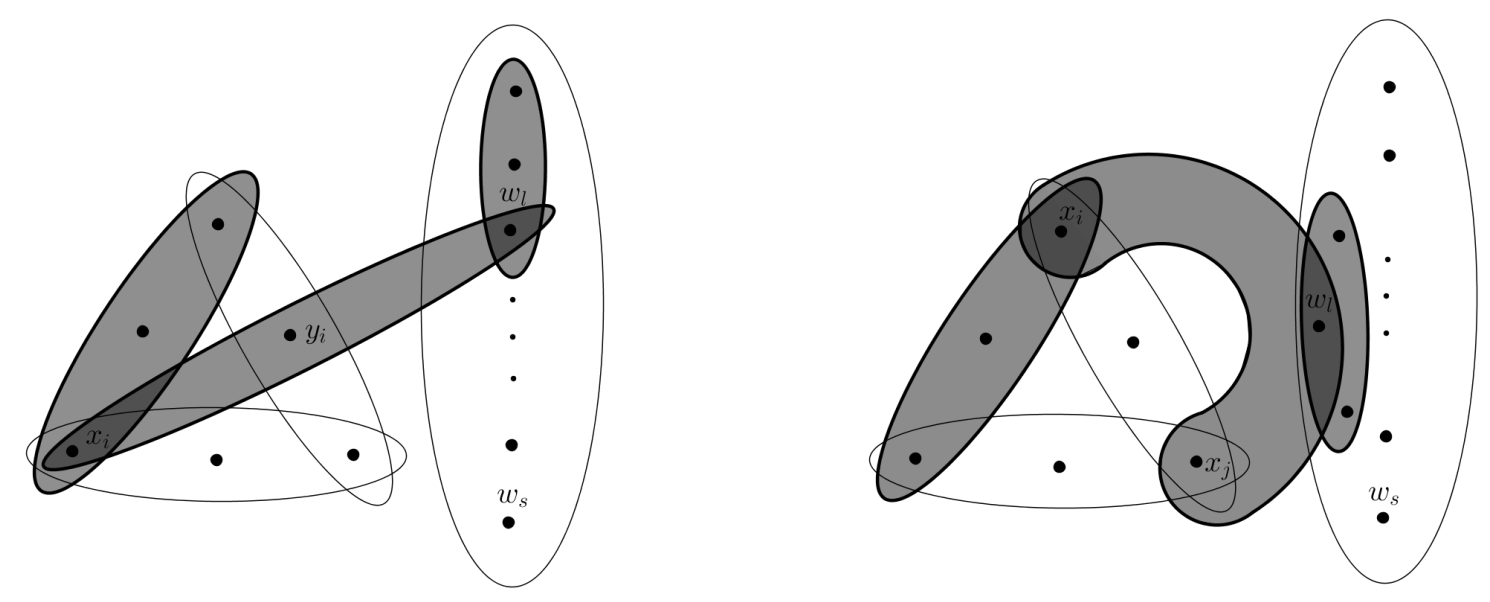

Figure 5: Illustration of Fact 2

Fact 2 ([9], Fact 6). If $e \in T, g \in\left(\begin{array}{c}W \\ 3\end{array}\right)$, and $e \cap g \neq \emptyset$, then $C \cup\{e\} \cup\{g\} \supset P_{3}^{3}$.

Similarly, two disjoint edges, one from $T_{1}$ and the other from $T$, would form a $P_{3}^{3}$ with an edge of $C$ (see Fig. 6).

Fact 3. If $e \in T_{1}, f \in T$, and $e \cap f=\emptyset$, then $C \cup\{e\} \cup\{f\} \supset P_{3}^{3}$.

We will also need the following simple consequence of König's Theorem.

Fact 4. In a $t \times s$ bipartite graph, where $t \leqslant s$, the largest possible number of edges not producing a matching of size $m+1, m \leqslant t$, is $s m$. 

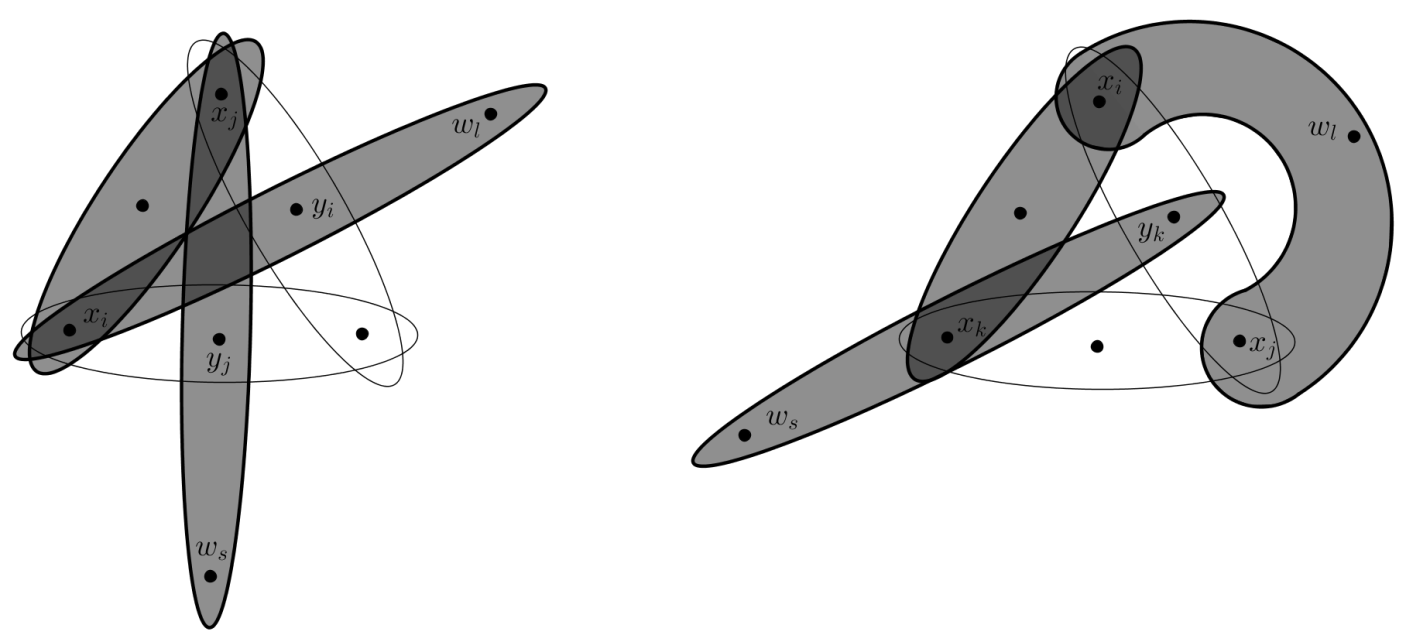

Figure 6: Illustration of Fact 3

Combining Fact 3 for $e, f \in T_{1}$ with Fact 4, we obtain the following corollary.

Corollary 3. For $s \geqslant 3$,

$$
\left|H \cap T_{1}\right| \leqslant s .
$$

Proof. Let $B$ be the auxiliary $3 \times s$ bipartite graph with vertex classes $\{1,2,3\}$ and $W$, where $\{i, w\}$ is an edge of $B$ if $\left\{x_{i}, y_{i}, w\right\} \in H$. Thus, $|B|=\left|H \cap T_{1}\right|$. By Fact 3 , there are no disjoint edges in $B$. Hence, by Fact 4 with $t=3$ and $m=1,|B| \leqslant s$.

Another consequence of Fact 3 has been already proved in [9]. We reproduce that proof for the sake of self-containment.

Proposition 1 ([9], Fact 4). For $s \geqslant 2$,

$$
|H \cap T| \leqslant 3 s .
$$

Proof. We have

$$
\left|T_{1}\right|=\left|T_{2}\right|=3 s .
$$

Construct an auxiliary bipartite graph $B=\left(T_{1}, T_{2} ; \mathcal{E}\right)$, where $\{e, f\} \in \mathcal{E}$ if $e \cap f=\emptyset$. It follows from Fact 3 that if $\{e, f\} \in \mathcal{E}$, then $|\{e, f\} \cap H| \leqslant 1$. Observe also that the graph $B$ is $(s-1)$-regular. Thus, by Hall's theorem, it has a perfect matching $M$. As at most one edge of each pair $\{e, f\} \in M$ can be in $H$, we infer that $|H \cap T| \leqslant 3 s$. 


\section{The lemmas}

To prove Theorem 1, we will need the following lemma which, with the notation of Section 2 , puts a cap on the total number of edges in the subgraphs $H[U]$ and $H(U, W)$, provided the latter is nonempty.

Lemma 1. For $s \geqslant 1$, if $H(U, W) \neq \emptyset$, then

$$
|H[U]|+|H(U, W)| \leqslant 13+\max \{3 s, 6\} .
$$

Proof. We begin by deducing upper bounds on $|H[U]|$ implied by the presence of an edge in

$$
H(U, W)=\left(H \cap T_{1}\right) \cup\left(H \cap T_{2}\right) .
$$

Assume first that $H \cap T_{1} \neq \emptyset$, say $\left\{x_{1}, y_{1}, w\right\} \in H \cap T_{1}$ for some $w \in W$. Let (cf. Fig. 2) $X_{1}=\left\{\left\{x_{1}, y_{2}, y_{3}\right\},\left\{x_{2}, y_{2}, y_{3}\right\},\left\{x_{3}, y_{2}, y_{3}\right\},\left\{x_{2}, y_{1}, y_{3}\right\},\left\{x_{3}, y_{1}, y_{2}\right\},\left\{x_{2}, x_{3}, y_{2}\right\},\left\{x_{2}, x_{3}, y_{3}\right\}\right\}$. One can easily check that if $H \cap X_{1} \neq \emptyset$, then $P_{3}^{3} \subseteq H$, a contradiction. Hence, $H[U] \subseteq$ $\left(\begin{array}{c}U \\ 3\end{array}\right) \backslash X_{1}$, and so,

$$
|H[U]| \leqslant\left|\left(\begin{array}{c}
U \\
3
\end{array}\right)\right|-\left|X_{1}\right|=20-7=13 .
$$

Similarly, if $e=\left\{x_{1}, x_{2}, w\right\} \in H \cap T_{2}$, then, by considering the set

$$
X_{2}=\left\{\left\{y_{1}, y_{2}, y_{3}\right\},\left\{x_{2}, y_{1}, y_{3}\right\},\left\{x_{3}, y_{1}, y_{3}\right\},\left\{x_{1}, y_{2}, y_{3}\right\},\left\{x_{3}, y_{2}, y_{3}\right\}\right\},
$$

one can show that

$$
|H[U]| \leqslant\left|\left(\begin{array}{c}
U \\
3
\end{array}\right)\right|-\left|X_{2}\right|=20-5=15 .
$$

In summary,

$$
H(U, W) \neq \emptyset \Longrightarrow|H[U]| \leqslant 15 .
$$

Therefore, if $|H(U, W)| \leqslant s$, then, with some margin,

$$
|H[U]|+|H(U, W)| \leqslant 15+s<13+\max \{3 s, 6\} .
$$

Consider now the case $|H(U, W)|>s$. Since by Fact 1, Proposition 1, and (2), for all $s \geqslant 1$ we have

$$
|H(U, W)| \leqslant \max \{3 s, 6\},
$$

it remains to show that (3) still holds. As explained above, this is the case when $H \cap T_{1} \neq \emptyset$. Otherwise, $\left|H \cap T_{2}\right|=|H(U, W)|>s$, and, since $|W|=s$, we infer that there exists a vertex $w \in W$ and two edges $e, f \in H \cap T_{2}$, both containing $w$. Then, necessarily, $|e \cap f \cap U|=1$. Say, $e \cap f \cap U=\left\{x_{1}\right\}$ (see Fig. 7). Consequently, to avoid a copy of $P_{3}^{3}$ in $H$, we must have $H \cap Y=\emptyset$, where

$$
Y=X_{2} \cup\left\{\left\{x_{2}, y_{2}, y_{3}\right\},\left\{x_{2}, y_{1}, y_{2}\right\},\left\{x_{3}, y_{1}, y_{2}\right\}\right\},
$$


and so,

$$
|H[U]| \leqslant\left|\left(\begin{array}{c}
U \\
3
\end{array}\right)\right|-|Y|=20-8=12,
$$

which is even better than (3). In conclusion, for all $s \geqslant 1$,

$$
|H(U, W)|>s \Longrightarrow|H[U]| \leqslant 13 .
$$

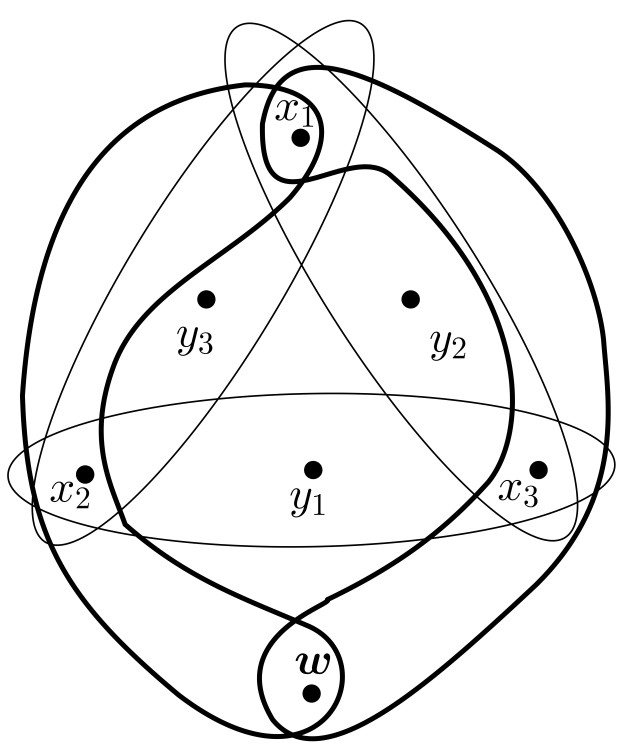

Figure 7: Illustration to the proof of Lemma 1

Putting together bounds (6) and (7) completes the proof of Lemma 1.

Since for $s \geqslant 2$ we have $\max \{3 s, 6\}=3 s$ and $|H[U]| \leqslant\left|\left(\begin{array}{c}U \\ 3\end{array}\right)\right|=20 \leqslant 14+3 s$, Lemma 1 has the following immediate consequence, true no matter whether $H(U, W)=\emptyset$ or not.

Corollary 4. For $s \geqslant 2$,

$$
|H[U]|+|H(U, W)| \leqslant 14+3 s .
$$

In the proof of Theorem 3 we will need a further improvement, under additional constraints, of the bound in Corollary 4.

Lemma 2. For $s \geqslant 3$, if $H(U, W) \neq \emptyset$, then

$$
|H[U]|+|H(U, W)| \leqslant 10+3 s .
$$


Proof. If $0<|H(U, W)| \leqslant s$, then, by (5),

$$
|H[U]|+|H(U, W)| \leqslant 15+s<10+3 s .
$$

Also, if $s<|H(U, W)| \leqslant 2 s$, then by $(7)$,

$$
|H[U]|+|H(U, W)| \leqslant 13+2 s \leqslant 10+3 s .
$$

For the rest of the proof we are assuming that

$$
|H(U, W)|=\left|H \cap T_{1}\right|+\left|H \cap T_{2}\right| \geqslant 2 s+1 .
$$

We are going to show that

$$
|H[U]| \leqslant 10 .
$$

Then the lemma will follow by Proposition 1.

Consider first the case when $H \cap T_{1}=\emptyset$. Then $\left|H \cap T_{2}\right| \geqslant 2 s+1$ and, thus, there must exist a vertex $w \in W$ such that all three edges $\left\{x_{i}, x_{j}, w\right\}, 1 \leqslant i<j \leqslant 3$, belong to $H$ (see Fig. 8).

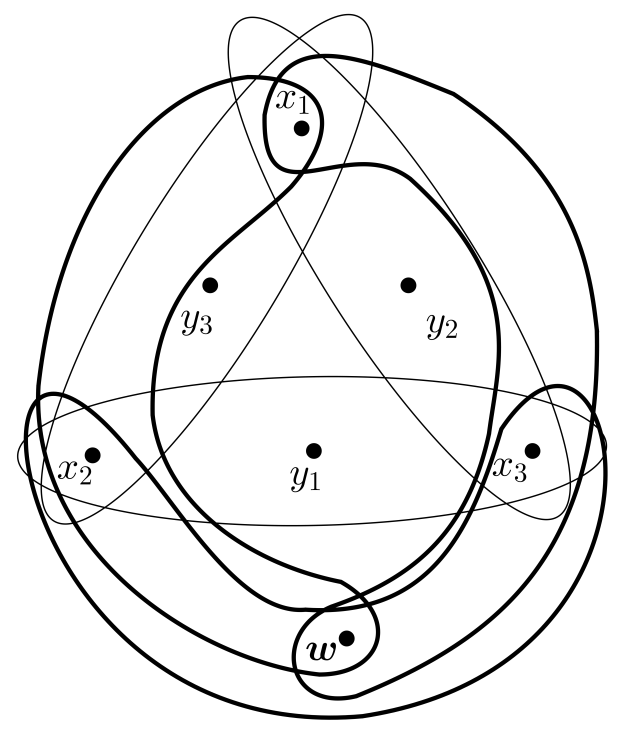

Figure 8: Illustration to the proof of Lemma 2: case $H \cap T_{1}=\emptyset$

But then, since $H$ is $P_{3}^{3}$-free, we have $H \cap Z_{1}=\emptyset$, where

$$
Z_{1}=\left\{\left\{y_{1}, y_{2}, y_{3}\right\},\left\{y_{i}, y_{j}, x_{k}\right\}: 1 \leqslant i<j \leqslant 3,1 \leqslant k \leqslant 3\right\}, \quad\left|Z_{1}\right|=10 .
$$

Thus, (8) holds.

Assume now that $H \cap T_{1} \neq \emptyset$. W.l.o.g., let $h^{\prime}=\left\{x_{1}, y_{1}, w^{\prime}\right\} \in H$, where $w^{\prime} \in W$, and distinguish two subcases. 
Subcase 1: For some $w^{\prime \prime} \in W, w^{\prime \prime} \neq w^{\prime}$, we have $h^{\prime \prime}=\left\{x_{1}, y_{1}, w^{\prime \prime}\right\} \in H$. By Fact 3, every edge of $H \cap T_{2}$ must intersect both, $h^{\prime}$ and $h^{\prime \prime}$. Thus, every edge of $H \cap T_{2}$ contains vertex $x_{1}$. Since, by (1), $\left|H \cap T_{1}\right| \leqslant s$, we infer that $\left|H \cap T_{2}\right|>s$. Consequently, there exists a vertex $w \in W$ with $\left\{x_{1}, x_{2}, w\right\}$ and $\left\{x_{1}, x_{3}, w\right\}$ belonging to $H$ (see Fig. 9 for the case when $\left.w=w^{\prime}\right)$.

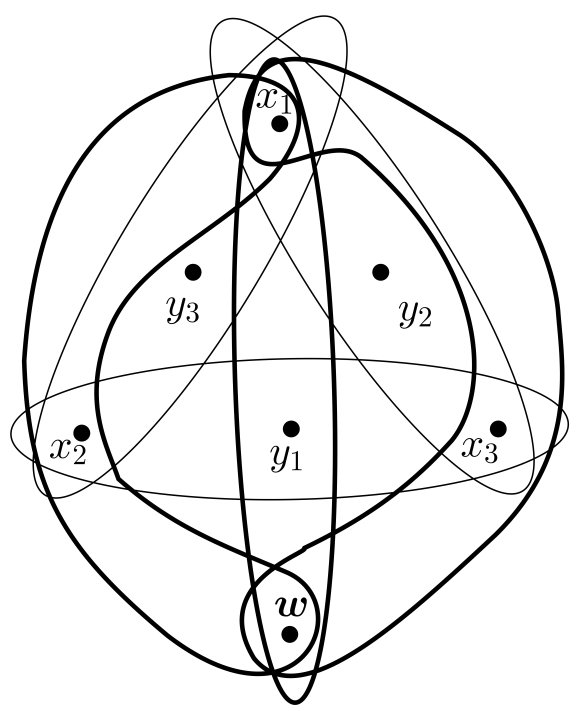

Figure 9: Illustration to the proof of Lemma 2: case $H \cap T_{1} \neq \emptyset$

But then $H \cap Z_{2}=\emptyset$, where

$$
Z_{2}=Y \cup X_{1}=Y \cup\left\{\left\{x_{2}, x_{3}, y_{2}\right\},\left\{x_{2}, x_{3}, y_{3}\right\}\right\}, \quad\left|Z_{2}\right|=10,
$$

and (8) holds.

Subcase 2: $H \cap T_{1} \subseteq\left\{\left\{x_{i}, y_{i}, w^{\prime}\right\}, 1 \leqslant i \leqslant 3\right\}$. Set $\left|H \cap T_{1}\right|=t, 1 \leqslant t \leqslant 3$. By Fact 3 , for every $i=1,2,3$, if $\left\{x_{i}, y_{i}, w^{\prime}\right\} \in H$ then $\left\{x_{j}, x_{k}, w\right\} \notin H$ for all $w \neq w^{\prime}$, where $\{j, k\}=\{1,2,3\} \backslash\{i\}$. Hence,

$$
\left|H \cap T_{2}\right| \leqslant t+(3-t) s,
$$

and, since $|H(U, W)|=\left|H \cap T_{1}\right|+\left|H \cap T_{2}\right| \geqslant 2 s+1 \geqslant 7$, we have $t \leqslant 2$. Moreover, for $t=2,2 s-1 \leqslant\left|H \cap T_{2}\right| \leqslant 2+s$ which forces $s=3$, and, consequently, $\left|H \cap T_{2}\right|=5$. This, in turn, implies the existence in $H$ of all three edges $\left\{x_{i}, x_{j}, w^{\prime}\right\}, 1 \leqslant i<j \leqslant 3$, as in the case $H \cap T_{1}=\emptyset$ discussed above, and, again (8) holds. Finally, if $t=1$, that is, $H \cap T_{1}=\left\{h^{\prime}\right\}$, then, letting $e^{\prime}=\left\{x_{2}, x_{3}, w^{\prime}\right\}$,

$$
\mid\left(H \cap\left(T_{2} \backslash\left\{e^{\prime}\right\}\right)|\geqslant| H \cap T_{2}|-1=| H \cap T \mid-2 \geqslant 2 s-1>s .\right.
$$


Consequently, there exists a vertex $w \in W$ belonging to two edges of $T_{2} \backslash\left\{e^{\prime}\right\}$. This means that regardless of whether or not $w=w^{\prime}$, the edges $\left\{x_{1}, x_{2}, w\right\}$ and $\left\{x_{1}, x_{3}, w\right\}$ both belong to $H$. As this is the same configuration as in Subcase 1 (cf. Fig. 9), the bound (8) holds again.

\section{Proofs of Theorems 1 and 3}

\subsection{Proof of Theorem 1}

This proof is by induction on $n$. Since $P_{3}^{3}$ contains 7 vertices, Theorem 1 is trivially true for $n \leqslant 6$. Although we begin the inductive step at $n=8$ only, our proof has the same logical structure for all $n \geqslant 7$. First note that both candidates for the extremal 3-graph, $H_{7}:=K_{6}^{3} \cup K_{1}$ for $n=7$ and $H_{n}:=S_{n}^{3}$ for $n \geqslant 8$, are $P_{3}^{3}$-free. We will be assuming that $H$ is a $P_{3}^{3}$-free 3 -graph, with $|V|=n,|H| \geqslant\left|H_{n}\right|$ and $H \neq H_{n}$. By Theorem 2, $H$ contains a copy $C$ of the triangle $C_{3}^{3}$. From that point on we will make our way toward an application of Lemma 1, leading to the inequality $|H|<\left|H_{n}\right|$, contradicting our assumption. Ultimately, we will show that no $P_{3}^{3}$-free 3 -graph on $n$ vertices and at least $\left|H_{n}\right|$ edges exists, except for $H_{n}$ itself, which is precisely the statement of Theorem 1 . Now come the details. Throughout, we keep the notation introduced in Section 2.

$\mathbf{n}=7$ (initial step). Let $H$ be a $P_{3}^{3}$-free 3-graph with $V(H)=V,|V|=n=7$ (thus, $s=1),|H| \geqslant 20$, and let $H \neq K_{6}^{3} \cup K_{1}$. Note that $20>\left(\begin{array}{c}7-1 \\ 2\end{array}\right)=15$ and so, by Theorem $2, H$ contains a copy $C$ of the triangle $C_{3}^{3}$. As $H \neq K_{6}^{3} \cup K_{1}$, we infer that $H(U, W) \neq \emptyset$. Hence, by Lemma 1,

$$
|H[U]|+|H(U, W)| \leqslant 13+\max \{3 s, 6\}=19<20,
$$

a contradiction.

$\mathbf{n} \geqslant 8$ (inductive step). Let $H$ be a $P_{3}^{3}$-free 3 -graph with $V(H)=V,|V|=n \geqslant 8$, $|H| \geqslant\left(\begin{array}{c}n-1 \\ 2\end{array}\right)$ and let $H \neq S_{n}^{3}$. By Theorem 2, $H$ contains a copy $C$ of the triangle $C_{3}^{3}$. By Corollary 4 , with $s=n-6$, we get

$$
|H|=|H[U]|+|H(U, W)|+|H[W]| \leqslant 14+3 s+\operatorname{ex}_{3}\left(s ; P_{3}^{3}\right) .
$$

Consequently, to complete the proof it remains to show that

$$
14+3 s+\operatorname{ex}_{3}\left(s ; P_{3}^{3}\right)<\left(\begin{array}{c}
n-1 \\
2
\end{array}\right)=\left(\begin{array}{c}
s+5 \\
2
\end{array}\right)
$$

that is, to show that

$$
\operatorname{ex}_{3}\left(s ; P_{3}^{3}\right)<\left(\begin{array}{c}
s+5 \\
2
\end{array}\right)-3 s-14=\left(\begin{array}{c}
s+2 \\
2
\end{array}\right)-5 \text {. }
$$


To this end, we rely on our induction's assumption, in particular, on the formula for $\operatorname{ex}_{3}\left(s ; P_{3}^{3}\right)$. For $s=\{2,3,4,5,6\}$ (equivalently, $n=\{8,9,10,11,12\}$ ), one can check by direct substitution that

$$
\operatorname{ex}_{3}\left(s ; P_{3}^{3}\right)=\left(\begin{array}{l}
s \\
3
\end{array}\right)<\left(\begin{array}{c}
s+2 \\
2
\end{array}\right)-5 .
$$

For $s=7(n=13)$,

$$
\operatorname{ex}_{3}\left(s ; P_{3}^{3}\right)=20<\left(\begin{array}{c}
7+2 \\
2
\end{array}\right)-5=31 .
$$

Finally, for $s \geqslant 8(n \geqslant 14)$,

$$
\operatorname{ex}_{3}\left(s ; P_{3}^{3}\right)=\left(\begin{array}{c}
s-1 \\
2
\end{array}\right)<\left(\begin{array}{c}
s-1 \\
2
\end{array}\right)+3 s-5=\left(\begin{array}{c}
s+2 \\
2
\end{array}\right)-5 .
$$

\subsection{Proof of Theorem 3}

Although not inductive, this proof is based on similar ideas to those used in the proof of Theorem 1, as well as on Theorem 1 itself. There is nothing to prove for $n=6$. From now on we will be assuming that $n \geqslant 7$, or equivalently, that $s \geqslant 1$ (again, we keep the notation introduced in Section 2).

Let $H$ be a $P_{3}^{3}$-free 3 -graph with $V(H)=V,|V|=n \geqslant 7$, containing a copy $C$ of the triangle $C_{3}^{3}$. Observe that if $H(U, W)=\emptyset$, then the only $P_{3}^{3}$-free, $n$-vertex 3 -graph with at least $20+\operatorname{ex}_{3}\left(n-6 ; P_{3}^{3}\right)$ edges consists of a copy of $K_{6}^{3}$ and a $P_{3}^{3}$-free extremal 3 -graph on $n-6$ vertices. Consequently, in order to prove Theorem 3, it is sufficient to show that if $H(U, W) \neq \emptyset$ then

$$
|H|<20+\operatorname{ex}_{3}\left(n-6 ; P_{3}^{3}\right) .
$$

Assume that $H(U, W) \neq \emptyset$. We split the set of vertices $W$ into two subsets (see Fig.10):

$$
W_{1}=\{w \in W: \text { there exists an edge } e \in H(U, W) \text { such that } w \in e\}
$$

and

$$
W_{2}=W \backslash W_{1} .
$$

Set $\left|W_{i}\right|=s_{i}, i=1,2$, where $s_{1}+s_{2}=s=n-6$. By Facts 1 and $2, H[W] \subset\left(\begin{array}{c}W_{2} \\ 3\end{array}\right)$. It turns out that all we need to show is that

$$
|H[U]|+|H(U, W)|<20+\operatorname{ex}_{3}\left(s_{1} ; P_{3}^{3}\right) .
$$

Indeed, by the subadditivity of $\operatorname{ex}_{3}(t ; F)$ as a function of $t$, we will then have

$$
|H|=|H[U]|+|H(U, W)|+|H[W]|<20+\operatorname{ex}_{3}\left(s_{1} ; P_{3}^{3}\right)+\operatorname{ex}_{3}\left(s_{2} ; P_{3}^{3}\right) \leqslant 20+\operatorname{ex}_{3}\left(s ; P_{3}^{3}\right) .
$$

For $1 \leqslant s_{1} \leqslant 2$, we apply Lemma 1 to the induced subhypergraph $H\left[U \cup W_{1}\right]$ to get

$$
|H[U]|+|H(U, W)| \leqslant 13+\max \left\{3 s_{1}, 6\right\}=19<20=20+\operatorname{ex}_{3}\left(s_{1} ; P_{3}^{3}\right) .
$$




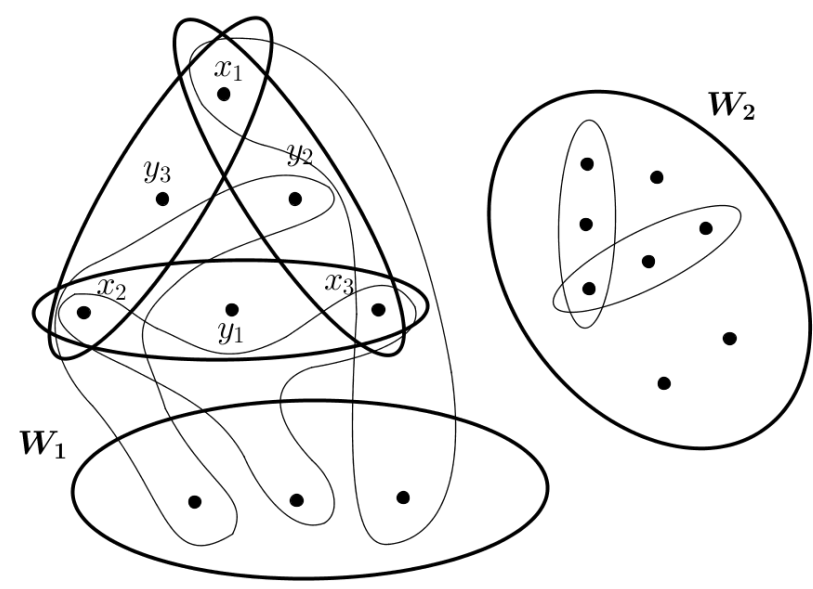

Figure 10: The division of the set $W$ into two subsets $W_{1}$ and $W_{2}$

Finally, assume that $s_{1} \geqslant 3$. By Lemma 2 applied to $H\left[U \cup W_{1}\right]$ and by Theorem 1 with $n:=s_{1}$, we conclude that

$$
|H[U]|+|H(U, W)| \leqslant 10+3 s_{1}<20+\operatorname{ex}_{3}\left(s_{1} ; P_{3}^{3}\right),
$$

where the verification of the last inequality is left to the reader.

Proof of Corollary 2. With the notation of the proof of Theorem 3, observe that the connectivity assumption implies that $W_{2}=\emptyset$. Thus, by Lemma 2

$$
|H|=|H[U]|+|H(U, W)| \leqslant 10+3(n-6)=3 n-8 .
$$

Moreover, the 3 -graph with vertex set $V$ and the edge set $\left(\left(\begin{array}{c}U \\ 3\end{array}\right) \backslash Z_{1}\right) \cup T_{2}$ contains $C_{3}^{3}$, is $P_{3}^{3}$-free and has $3 n-8$ edges.

\section{Conditional Turán numbers}

Inspired by Theorem 3, in this final section we discuss some restricted versions of Turán numbers. We begin with a general definition of the conditional Turán numbers.

Given an integer $n$, a family of $k$-graphs $\mathcal{F}$, and a family of $\mathcal{F}$-free $k$-graphs $\mathcal{G}$, let $\operatorname{ex}_{k}(n ; \mathcal{F} \mid \mathcal{G})$ be the largest number of edges in an $n$-vertex $\mathcal{F}$-free $k$-graph $H$ such that $H \supseteq G$ for some $G \in \mathcal{G}$. If $\mathcal{F}=\{F\}$ or $\mathcal{G}=\{G\}$, we will simply write $\operatorname{ex}_{k}(n ; F \mid \mathcal{G})$, $\operatorname{ex}_{k}(n ; \mathcal{F} \mid G)$, or $\operatorname{ex}_{k}(n ; F \mid G)$, respectively.

Of course, we have $\operatorname{ex}_{k}(n ; \mathcal{F} \mid \mathcal{G}) \leqslant \operatorname{ex}_{k}(n ; \mathcal{F})$. For instance, comparing Theorems 1 and 3 , we see that for $n \geqslant 14$

$$
\operatorname{ex}_{3}\left(n ; P_{3}^{3}\right)-\operatorname{ex}_{3}\left(n ; P_{3}^{3} \mid C_{3}^{3}\right)=6 n-47 .
$$


In view of the equality $\operatorname{ex}_{3}\left(n ; P_{3}^{3}\right)=\operatorname{ex}_{3}\left(n ; C_{3}^{3}\right)$ (for $\left.n \geqslant 8\right)$, it would be also interesting to calculate the reverse conditional Turán number, namely $\operatorname{ex}_{3}\left(n ; C_{3}^{3} \mid P_{3}^{3}\right)$. For $n \geqslant 7$, consider a 3-graph $H(n ; C \mid P)$ consisting of an edge $\{x, y, z\}$ and all edges of the form $\{x, y, w\}, w \neq z$, and $\left\{z, w^{\prime}, w^{\prime \prime}\right\}$, where $\left\{w^{\prime}, w^{\prime \prime}\right\} \cap\{x, y\}=\emptyset$ (see Fig. 11).

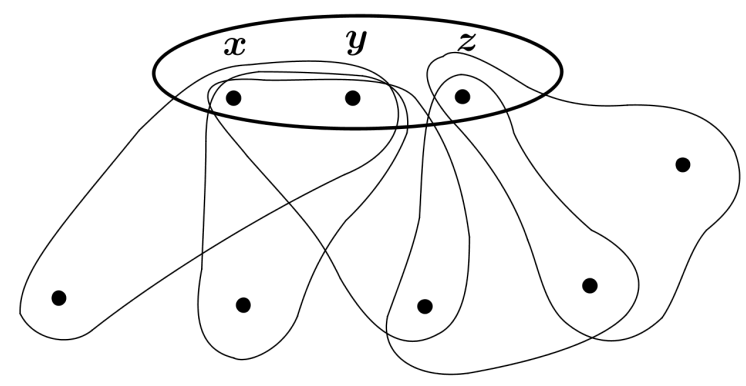

Figure 11: Part of the 3-graph $H(n ; C \mid P)$

Note that $P_{3}^{3} \subseteq H(n ; C \mid P) \nsupseteq C_{3}^{3}$ and thus

$$
\operatorname{ex}_{3}\left(n ; C_{3}^{3} \mid P_{3}^{3}\right) \geqslant|H(n ; C \mid P)|=1+(n-3)+\left(\begin{array}{c}
n-3 \\
2
\end{array}\right)=\left(\begin{array}{c}
n-2 \\
2
\end{array}\right)+1 .
$$

So, again a conditional Turán number, though not yet determined, is going to be not much smaller than its unconditional counterpart. This is not a coincidence. In fact, we have the following observation.

Proposition 2. If $\mathcal{F}$ consists of connected $k$-graphs only and neither $\mathcal{F}$ nor $\mathcal{G}$ depends on $n$, then

$$
\operatorname{ex}_{k}(n ; \mathcal{F} \mid \mathcal{G}) \sim \operatorname{ex}_{k}(n ; \mathcal{F})
$$

Proof. By considering a disjoint union of any $G \in \mathcal{G}$ and any extremal $\mathcal{F}$-free graph on $n-|V(G)|$ vertices, we have

$$
\operatorname{ex}_{k}(n-|V(G)| ; \mathcal{F})+|E(G)| \leqslant \operatorname{ex}_{k}(n ; \mathcal{F} \mid \mathcal{G}) \leqslant \operatorname{ex}_{k}(n ; \mathcal{F})
$$

Moreover, by removing $g=|V(G)|$ vertices of smallest degrees from an extremal $\mathcal{F}$-free $k$-graph on $n$ vertices, we infer that

$$
\operatorname{ex}_{k}(n-g ; \mathcal{F}) \geqslant \operatorname{ex}_{k}(n ; \mathcal{F})\left(1-\frac{k g}{n-g}\right)
$$




\subsection{Nontrivial intersecting families}

For disconnected $F$, conditioning on the presence of specified subhypergraphs may cause a Turán number drop significantly. A prime example of this phenomenon is the celebrated Erdős-Ko-Rado Theorem on the maximum size of intersecting families. It asserts that for $n \geqslant 2 k$, with $M_{2}^{k}$ standing for a pair of disjoint $k$-sets, $\operatorname{ex}_{k}\left(n ; M_{2}^{k}\right)=\left(\begin{array}{c}n-1 \\ k-1\end{array}\right)$, and, for $n \geqslant 2 k+1, \operatorname{Ex}_{k}\left(n ; M_{2}^{k}\right)=\left\{S_{n}^{k}\right\}$. It was thus quite natural to ask what is the largest number of edges in an $n$-vertex $M_{2}^{k}$-free $k$-graph which is not a star (the so called nontrivial intersecting family). Hilton and Milner [8] proved that the answer to this question is $\left(\begin{array}{l}n-1 \\ k-1\end{array}\right)-\left(\begin{array}{c}n-k-1 \\ k-1\end{array}\right)+1$ (see [5] for a short proof).

For $k=3$, it can be checked that an intersecting triple system is not a star if, and only if, it contains either the triangle $C_{3}^{3}$ or the 3 -graph

$$
F_{5}=(\{a, b, c, d, e\},\{\{a, b, c\},\{c, d, e\},\{e, a, b\}\}),
$$

or the clique $K_{4}^{3}$. From this perspective, the above strengthening of the E-K-R Theorem, due to Hilton and Milner, can be reformulated, for $k=3$, as

$$
\operatorname{ex}_{3}\left(n ; M_{2}^{3} \mid\left\{C_{3}^{3}, F_{5}, K_{4}^{3}\right\}\right)=3 n-8 .
$$

Hence, for $\mathcal{F}=\left\{M_{2}^{3}\right\}$, a conditional Turán number can be much smaller than the unconditional one (linear vs. quadratic function of $n$.)

\subsection{Second order Turán numbers}

The Turán numbers for $P_{3}^{k}$ and $C_{3}^{k}$ reveal a whole lot of similarity to the E-K-R Theorem. Indeed, restricting just to the case $k=3$, we have, for $n \geqslant 8$,

$$
\operatorname{ex}_{3}\left(n ; P_{3}^{3}\right)=\operatorname{ex}_{3}\left(n ; C_{3}^{3}\right)=\operatorname{ex}_{3}\left(n ; M_{2}^{3}\right)=\left(\begin{array}{c}
n-1 \\
2
\end{array}\right)
$$

and

$$
\operatorname{Ex}_{3}\left(n ; P_{3}^{3}\right)=\operatorname{Ex}_{3}\left(n ; C_{3}^{3}\right)=\operatorname{Ex}_{3}\left(n ; M_{2}^{3}\right)=\left\{S_{n}^{3}\right\} .
$$

Therefore, like in the E-K-R case, one might ask for the largest size of a nontrivial $P_{3}^{3}$-free (or $C_{3}^{3}$-free) 3-graph, that is, one which is not a star.

Let us generalize this question. Suppose that for some $n$ and $F$, we have $\operatorname{Ex}_{k}(n ; F)=$ $\{H(n ; F)\}$, that is, there is a unique (up to isomorphism) extremal $F$-free $n$-vertex $k$-graph $H(n ; F)$. Let $\overline{\operatorname{ex}}_{k}(n ; F)$ be the largest number of edges in an $F$-free $n$-vertex $k$-graph $H$ such that $H \nsubseteq H(n ; F)$. (Besides, the nontrivial intersecting families, a version of this parameter has been studied already for cliques in graphs, see [1], where the classical Turán number $\operatorname{ex}_{2}\left(n ; K_{t}\right)$ was restricted to non- $(t-1)$-partite graphs).

For $P_{3}^{3}$ and $C_{3}^{3}$, the defined above 'second order' Turán numbers turn out to coincide with the corresponding conditional numbers with respect to $M_{2}^{3}$, a pair of disjoint edges. 
Proposition 3. We have

$$
\overline{\operatorname{ex}}_{3}\left(n ; P_{3}^{3}\right)=\operatorname{ex}_{3}\left(n ; P_{3}^{3} \mid M_{2}^{3}\right) \quad \text { for } n \geqslant 11
$$

and

$$
\overline{\operatorname{ex}}_{3}\left(n ; C_{3}^{3}\right)=\operatorname{ex}_{3}\left(n ; C_{3}^{3} \mid M_{2}^{3}\right) \quad \text { for } n \geqslant 8 \text {. }
$$

Proof. Observe that, for each $F \in\left\{P_{3}^{3}, C_{3}^{3}\right\}$

$$
\overline{\operatorname{ex}}_{3}(n ; F)=\max \left[\operatorname{ex}_{3}\left(n ; F \mid M_{2}^{3}\right), \overline{\operatorname{ex}}_{3}\left(n ;\left\{F, M_{2}^{3}\right\}\right)\right]
$$

and

$$
\overline{\operatorname{ex}}_{3}\left(n ;\left\{F, M_{2}^{3}\right\}\right) \leqslant \overline{\operatorname{ex}}_{3}\left(n ; M_{2}^{3}\right) \stackrel{(9)}{=} 3 n-8 .
$$

Now, consider the following constructions for $n \geqslant 6$. Let $H(n ; P \mid M)$ be the union of a clique $K_{4}^{3}$ and a full star $S_{n-3}^{3}$ whose center is located at one of the vertices of the clique, but which otherwise is vertex-disjoint from the clique (see Fig. 12). Then $M_{2}^{3} \subseteq H(n ; P \mid M) \nsupseteq P_{3}^{3}$ and so

$$
\operatorname{ex}_{3}\left(n ; P_{3}^{3} \mid M_{2}^{3}\right) \geqslant|H(n ; P \mid M)|=\left(\begin{array}{c}
n-4 \\
2
\end{array}\right)+4 \geqslant 3 n-8
$$

for $n \geqslant 11$, which, in turn, implies that

$$
\overline{\operatorname{ex}}_{3}\left(n ; P_{3}^{3}\right)=\operatorname{ex}_{3}\left(n ; P_{3}^{3} \mid M_{2}^{3}\right) .
$$

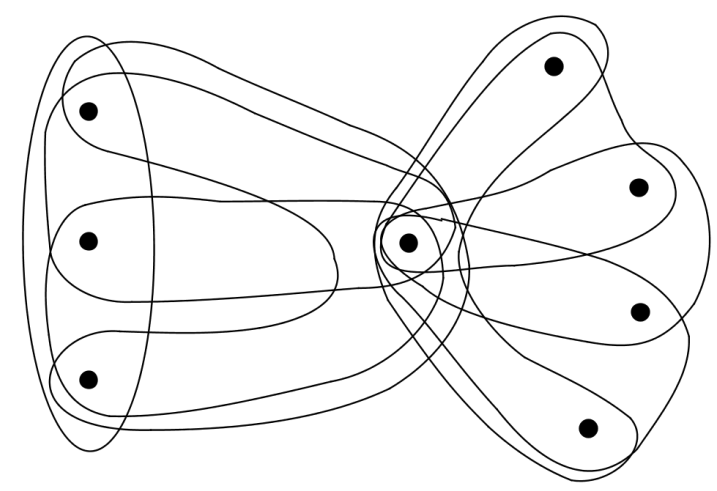

Figure 12: Part of the 3-graph $H(n ; P \mid M)$

To prove the second equation, we use again the 3-graph $H(n ; C \mid P)$ constructed earlier in this section. Since $M_{2}^{3} \subset P_{3}^{3}$,

$$
\operatorname{ex}_{3}\left(n ; C_{3}^{3} \mid M_{2}^{3}\right) \geqslant \operatorname{ex}_{3}\left(n ; C_{3}^{3} \mid P_{3}^{3}\right) \geqslant|H(n ; C \mid P)| \geqslant\left(\begin{array}{c}
n-2 \\
2
\end{array}\right)+1 \geqslant 3 n-8
$$


for $n \geqslant 8$, and thus, we also have

$$
\overline{\operatorname{ex}}_{3}\left(n ; C_{3}^{3}\right)=\operatorname{ex}_{3}\left(n ; C_{3}^{3} \mid M_{2}^{3}\right) .
$$

\section{Open problems and remarks}

It would be interesting to verify the following conjecture in which we express our belief that these conditional Turán numbers are, indeed, determined by the above described constructions.

Conjecture 1. With a possible exception of some small values of $n$,

$$
\begin{gathered}
\operatorname{ex}_{3}\left(n ; P_{3}^{3} \mid M_{2}^{3}\right)=\left(\begin{array}{c}
n-4 \\
2
\end{array}\right)+4 \\
\operatorname{ex}_{3}\left(n ; C_{3}^{3} \mid M_{2}^{3}\right)=\operatorname{ex}_{3}\left(n ; C_{3}^{3} \mid P_{3}^{3}\right)=\left(\begin{array}{c}
n-2 \\
2
\end{array}\right)+1 .
\end{gathered}
$$

Remark 2. We intend to address the first conjecture in a forthcoming paper [10]. If true, it would imply that (again, except for some small $n$ )

$$
\operatorname{ex}_{3}\left(n ; C_{3}^{3} \mid M_{2}^{3}\right)=\operatorname{ex}_{3}\left(n ; C_{3}^{3} \mid P_{3}^{3}\right) .
$$

Indeed, if $\operatorname{ex}_{3}\left(n ; P_{3}^{3} \mid M_{2}^{3}\right) \leqslant\left(\begin{array}{c}n-4 \\ 2\end{array}\right)+4$, then

$$
\operatorname{ex}_{3}\left(n ; C_{3}^{3} \mid P_{3}^{3}\right) \geqslant|H(n ; C \mid P)|=\left(\begin{array}{c}
n-2 \\
2
\end{array}\right)+1 \geqslant\left(\begin{array}{c}
n-4 \\
2
\end{array}\right)+4 \geqslant \operatorname{ex}_{3}\left(n ; P_{3}^{3} \mid M_{2}^{3}\right) .
$$

Thus,

$$
\begin{aligned}
\operatorname{ex}_{3}\left(n ; C_{3}^{3} \mid M_{2}^{3}\right) & =\max \left[\operatorname{ex}_{3}\left(n ; C_{3}^{3} \mid\left\{M_{2}^{3}, P_{3}^{3}\right\}\right), \operatorname{ex}_{3}\left(n ;\left\{C_{3}^{3}, P_{3}^{3}\right\} \mid M_{2}^{3}\right)\right] \\
& \leqslant \max \left[\operatorname{ex}_{3}\left(n ; C_{3}^{3} \mid P_{3}^{3}\right), \operatorname{ex}_{3}\left(n ; P_{3}^{3} \mid M_{2}^{3}\right)\right]=\operatorname{ex}_{3}\left(n ; C_{3}^{3} \mid P_{3}^{3}\right),
\end{aligned}
$$

which, together with the obvious inverse inequality, implies (10).

Remark 3. Conditional Turán numbers defined in this paper may be a useful tool in determining the corresponding Ramsey numbers. For instance, in [9] it has been shown that $R\left(P_{3}^{3} ; 3\right)=9$ by observing that if the triples of the clique $K_{9}^{3}$ are 3 -colored than at least one color appears on more than 28 edges, or all three colors appear each on precisely 28 edges. In either case, Theorem 1 implies that there must be a monochromatic copy of $P_{3}^{3}$ (in the latter case, because one cannot partition $K_{9}^{3}$ into 3 stars). For more than 3 colors this simple approach does not work any more, but instead one needs to look at the numbers $\overline{\operatorname{ex}}_{3}\left(n ; P_{3}^{3}\right)$ and beyond (see [10] and [12] for results on refined Turán numbers for $P_{3}^{3}$ leading to the determination of $R\left(P_{3}^{3} ; r\right)$ for $\left.4 \leqslant r \leqslant 9\right)$. 


\section{Acknowledgments}

We are very grateful to both referees for their valuable remarks which have led to a better presentation of our results. In particular, Reviewer A drew to our attention that Theorem 1 , for large $n$, can be derived from a result in [11]. Moreover, the same reviewer provided us with an alternative proof of Theorem 1, valid for all $n$, which instead of Theorem 2 is based on the Erdös-Ko-Rado Theorem. We chose, however, not to include that proof in our paper.

\section{References}

[1] K. Amin, J. Faudree, R. J. Gould, E. Sidorowicz, On the non- $(p-1)$-partite $K_{p}$-free graphs, Discuss. Math. Graph Theory, 33:9-23, 2013.

[2] N. Bushaw, N. Kettle, Turán numbers for forests of paths in hypergraphs, SIAM Journal on Discrete Mathematics, 28(2):711-721, 2014.

[3] R. Csákány, J. Kahn, A homological Approach to Two Problems on Finite Sets, Journal of Algebraic Combinatorics, 9:141-149, 1999.

[4] P. Erdős, A problem on independent r-tuples, Ann. Univ. Sci. Budapest. Eötvös Sect. Math., 8:93-95, 1965.

[5] P. Frankl, Z. Füredi, Non-trivial Intersecting Families Journal of Combinatorial Theory, Ser. A, 41:150-153, 1986.

[6] P. Frankl, Z. Füredi, Exact solution of some Turán-type problems, Journal of Combinatorial Theory, Ser. A, 45:226-262, 1987.

[7] Z. Füredi, T. Jiang, R. Seiver, Exact solution of the hypergraph Turán problem for k-uniform linear paths, Combinatorica, 34(3):299-322, 2014

[8] A. J. W. Hilton, E. C. Milner, Some intersection theorems for systems of finite sets, Quart. J. Math. Oxford Ser., 18(2):369-384, 1967.

[9] E. Jackowska, The 3-color Ramsey number for a 3-uniform loose path of length 3, Australasian J. Combin., 63(2):314-320, 2015.

[10] E. Jackowska, J. Polcyn, A Ruciński, Multicolor Ramsey numbers and restricted Turán numbers for the loose 3-uniform path of length three, submitted.

[11] A. Kostochka, D. Mubayi, J. Verstraëte, newblock Turán problems and shadows I: Paths and cycles, Journal of Combinatorial Theory, Ser. A, 129: 57-79, 2015.

[12] J. Polcyn, A Ruciński, Refined Turán numbers and Ramsey numbers for the loose 3-uniform path of length three, submitted. 